\title{
ANÁLISE DE RISCO FAMILIAR NA ESTRATÉGIA SAÚDE DA FAMÍLIA: UMA VIVÊNCIA COMPARTILHADA ENTRE PRECEPTORES, DISCENTES E AGENTES COMUNITÁRIOS DE SAÚDE
}

\author{
Ricardo Henrique Vieira De Melo
}

Aluno do Programa de Mestrado Profissional em Saúde da Família - RENASF / UFRN, Especialista em Saúde da Família - NESC / UFRN, Cirurgião-Dentista da Estratégia Saúde da Família do Município de Natal / RN, E-mail: ricardohvm@ gmail.com

\section{Rosana Lúcia Alves de Vilar}

Doutora em Ciências Sociais - UFRN, Docente do Departamento de Enfermagem / NESC / UFRN, Docente do Programa de Mestrado Profissional em Saúde da Família -

RENASF/UFRN, E-mail: rosanaalvesrn@gmail.com

\section{Antõnio Fabiano Ferreira}

Monitor do PET Saúde, Acadêmico do Curso de Odontologia / UFRN, E-mail: fabianoferreiraodonto@hotmail.com

\section{Eduardo Judene da Silva Pereira}

Monitor do PET Saúde, Acadêmico do Curso de Medicina / UFRN, E-mail: eduardo.med.ufrn@gmail.com

Naiara Estefânia Alves Carneiro

Monitora do PET Saúde, Acadêmica do Curso de Serviço Social / UFRN,

E-mail: naiara_eac@hotmail.com

\section{Nathália Gabrielle Henrique Bezerra de Freitas}

Monitora do PET Saúde, Acadêmica do Curso de Medicina / UFRN, E-mail: nathaliaghbfreitas@gmail.com

\section{José Diniz Junior}

Doutor em Medicina, Docente do Departamento de Cirurgia / UFRN, Tutor do PET Saúde

- Grupo Tutorial Cidade Praia, E-mail: dinizotorrino@gmail.com

\section{RESUMO}

O estudo do risco familiar deve ser pautado nas teias dos significados sociais que o próprio homem tece e destece no cotidiano, em meio ao que extravasa das casualidades e nas relações e determinações sociais. A Estratégia Saúde da Família carreia o dilema da priorização da atenção às famílias de maior vulnerabilidade biológica e social. Este artigo tem o objetivo de relatar os resultados da validação de um instrumento utilizado para estratificação de risco familiar para priorização de visitas domiciliares.Foram utilizados registros de observações em diário de campo e dos portfólios de aprendizagem dos alunos dos cursos da área de saúde da UFRN. O estudo foi realizado durante o primeiro semestre letivo do ano de 2013. Inicialmente foram realizadas rodas de leitura entre o Grupo Tutorial do PET-Saúde, Profissionais do NASF, Agentes Comunitários de Saúde e demais trabalhadores da unidade. Em seguida, a escala foi aplicada em regime de mutirão por grupos de trabalho compostos por Agentes de Saúde e Monitores supervisionados por Preceptores. Foram analisadas 1904 fichas "A" do Sistema de Informação da Atenção Básica. Os escores gerais foram semelhantes, entretanto as micro áreas compostas por conjuntos habitacionais mostraram menor risco quando comparadas com as micro áreas constituídas por loteamentos, vilas residenciais e terrenos baldios. Foram observadas médias de 5,8\% (equipe 046) e 6,7\% (equipe 048) de famílias classificadas como de alto 
risco. A escala foi pertinente, entretanto é necessário maior investimento em outras ferramentas de análise das condições de vulnerabilidade não contempladas pela escala, tais como: condições de higiene, poluição local, condutas pessoais de alimentação, sedentarismo, aspectos relacionais, interpessoais e criminais. O estudo proporcionou aos discentes uma melhor compreensão da interface entre saúde, doença e cuidado, durante o processo de ensino-aprendizagem, multiplicando as frentes de trabalho em direção à discussão e construção de Projetos Terapêuticos Singulares compartilhados pela tocada interdisciplinar.

Palavras-chaves: Estratégia Saúde da Família, Risco Familiar, Vulnerabilidade Social.

\title{
RISK ANALYSIS IN THE FAMILY HEALTH STRATEGY: AN EXPERIENCE SHARED BETWEEN PRECEPTORS, STUDENTS AND COMMUNITY HEALTH AGENTS
}

\begin{abstract}
The study of familial risk should be based on the social webs of meaning which the man weaves and unweaves in everyday life, in the midst of going beyond the casualties and relationships and social determinations. The Family Health Strategy carries the dilemma of prioritizing attention to families of greater biological and social vulnerability. This article aims to report the results of validating a tool used for risk stratification for prioritizing family visits. Records on daily field observations and portfolios of student learning courses healthcare of UFRN were used. The study was conducted during the first halfof the year 2013 through weekly meetings. Initially reading circles were held between the tutorial group of PET - Health, NASF's professionals, and community health workers. Then, the scale was applied under a joint effort by working groups composed by health agents and monitors supervised by preceptors. Were analyzed 1904 records "A" from the Information System of Primary Care. Overall scores were similar, however the micro areas consist of housing showed lower risk compared with the micro areas consist of lots, residential villas and wastelands. Means were observed in 5.8\% (046 staff) and 6.7\% (Team 048) of families classified as high risk. The scale was relevant, however it is necessary to invest more in others conditions of vulnerability not covered by the scale, such as hygiene, local pollution, conduct personal diet, physical inactivity, relational aspects, interpersonal and criminal. The experience provided the students with a better understanding of the interface between health, illness and care during the teaching-learning process, multiplying the work fronts toward discussion and construction of individualized therapeutic projects shared by interdisciplinary played.
\end{abstract}

Palavras-chaves: Family Health Strategy, Family Risk, Social Vulnerability.

\section{ANÁLISE DE RISCO FAMILIAR NA ESTRATÉGIA SAÚDE DA FAMÍLIA: UMA VIVÊNCIA COMPARTILHADA ENTRE PRECEPTORES, DISCENTES E AGENTES COMUNITÁRIOS DE SAÚDE}

\section{INTRODUÇÃO}

O Sistema Único de Saúde (SUS) tem como princípios fundamentais a universalidade, a integralidade e a equidade, que devem ser considerados na Atenção Básica, cuja estratégia 
prioritária para a organização é a Saúde da Família, que baseia suas atividades no diagnóstico situacional dirigido à família e comunidade.

A visita domiciliar figura como uma das importantes ações da Estratégia Saúde da Família e é um dos instrumentos utilizados na atenção à saúde que aproxima os profissionais do contexto sócio familiar contribuindo sobremaneira para consolidação de vínculos. Entretanto, traz consigo um dilema com relação a quem visitar primeiro e como priorizar famílias de maior risco sem perder a qualidade da atenção às famílias de menor risco.

Neste contexto surgiu, em 2003, a escala de risco familiar proposta por Coelho e Savassi (popularmente conhecida por Escala de Coelho), que revisa os critérios de preenchimento da Ficha A do Sistema de Informação da Atenção Básica (SIAB), com atenção especial às sentinelas de risco.

O estudo do risco familiar deve ser pautado nas teias dos significados sociais que o próprio homem tece e destece no cotidiano, em meio ao que extravasa das casualidades e nas relações e determinações sociais. Consonante ao princípio de equidade, a Estratégia Saúde da Família precisa priorizar a atenção às famílias de maior vulnerabilidade biológica e social.

A família constitui um sistema aberto, dinâmico e complexo, cujos membros pertencem a um mesmo contexto social e dele compartilham. É o lugar do reconhecimento da diferença e do aprendizado quanto ao unir-se e separar-se, é a sede das primeiras trocas afetivoemocionais e da construção da identidade. A estrutura das relações se manifesta através da comunicação dos papéis, normas e regras estabelecidos destro deste sistema.

Neste aspecto, ao longo do ciclo de vida, a família precisa de uma série de ajustes para lidar com os problemas que surgem para cumprir o seu papel de construção de sujeitos livres e autônomos. $\mathrm{O}$ foco na família, que é uma instância intermediária entre o indivíduo e a comunidade, deverá ser o horizonte a ser buscado nas estratégias de saúde da atenção primária.

A opção por uma abordagem no microssistema familiar dos agrupamentos humanos implica em um reconhecimento de que é no seio familiar que o ciclo da vida floresce, onde o indivíduo inicia a sua formação e começa a estabelecer suas concepções sobre o mundo que o cerca tecendo as estratégias que acredita que sejam pertinentes para sua relação com a sociedade.

As demandas de pessoas e grupos vulneráveis vinculados às equipes de saúde influenciam: o acesso aos serviços de saúde e suas formas de organização, a magnitude do vínculo entre usuários e profissionais, o tipo real de acolhimento, as ações preconizadas para a prevenção e o controle de agravos e os recursos sociais existentes no território.

Na visão de Bastos e Franco (2007), a opção por uma estratégia que aborda o coletivo familiar que está vinculado a uma equipe de referência local reproduz concepções na pluralidade dos membros familiares e nas experiências diversificadas das relações de convivência, conflitos, consensos, que acumulam saberes, hábitos e costumes.

Nesta lógica, o planejamento da assistência deve ser centrado nas necessidades da comunidade residente no território de abrangência da Unidade de Saúde da Família e seus objetivos devem se aproximar ao máximo das singularidades locais. Assim a intervenção através de Visitas Domiciliares favorece o exercício da integralidade junto ao indivíduo inserido num cotidiano e pertencente a uma família. 
Através de visitas a equipe passa a conhecer melhor as condições de vida e saúde da comunidade, e os recursos de sobrevivência disponíveis, propiciando a corresponsabilidade do indivíduo ou família, enquanto sujeitos de sua própria saúde, a decidir junto com a equipe multiprofissional, sobre os problemas e agravos identificados.

Para isso é necessário o entendimento e o respeito sobre o contexto de vida singular de cada comunidade, sua história, seus costumes e suas dinâmicas de relações familiares, buscando eliminar a postura de superioridade ou o autoritarismo da mera reprodução do modelo centrado apenas na consulta, no remédio ou no procedimento, agora no domicílio.

Considerando a maioria das capitais brasileiras, as equipes de saúde se encontram sobrecarregadas com uma cobertura populacional que ultrapassa os parâmetros recomendados nacionalmente. A Unidade de Saúde da Família de Cidade Praia, por exemplo, possuiaproximadamente 2.000 famílias, totalizando mais ou menos 7.000 pessoas para suas duas equipes constituídas.

Com essa superlotação, passou a ser um dilema estabelecer qual família se deveria visitar primeiro. Como será possível privilegiar algumas famílias de maior risco sem desprender o olhar vigilante às famílias de menor risco? Como aplicar localmente o princípio da equidade sem descuidar da totalidade? Há algo inovador a contribuir com este debate? Como enfatizar a propagação da discussão? Qual a relevância desta discussão para os cenários de prática?

A fim de refletir sobre estas questões, no entanto sem a intenção de esgotá-las, o presente artigo tem por objetivo compartilhar a sistematização de uma experiência de uso e validação de um instrumento para classificação de risco familiar no escopo da Estratégia Saúde da Família, vivenciada por tutores, preceptores, discentes e demais profissionais das equipes de saúde e do Núcleo de Apoio à Saúde da Família, ocupantes de um lugar privilegiado indutor de mudanças e criador de práxis, no mar de possibilidades entre a academia, serviços e comunidade.

Neste contexto a aplicação da Escala de Coelho teve como objetivos: Conhecer os graus de risco das famílias adscritas; Propor o uso deste instrumento como rotina no processo de trabalho local; e Contribuir com o compromisso ético de fortalecimento da atenção básica como coordenadora do cuidado.

\section{PASSEIO TEÓRICO}

A visita domiciliar como qualquer outra atividade precisa ser planejada e sistematizada para se configurar como parte do arsenal de atenção na Estratégia Saúde da Família, onde se torna imprescindível a valorização do trabalho em equipe, a troca de informações e conhecimentos sobre o paciente, o conhecimento das competências comuns e específicas de todos os profissionais envolvidos visando uma atenção integral com resolutividade adequada.

Ayres et al (2012), apreendendo o sentido prático da integralidade, adota quatros eixos interligados na organização da atenção à saúde: Necessidades (qualidade e natureza da escuta, acolhimento e resposta às demandas de atenção à saúde); Finalidades (graus e modos de integração entre as ações de promoção, prevenção, tratamento e recuperação/reinserção, todas em sinergismo); Articulações (saberes interdisciplinares, equipes multiprofissionais, ações intersetoriais das estratégias de atenção à saúde); 
Interações (qualidade e natureza das interações intersubjetivas do cotidiano das práticas de cuidado).

Na realidade, para Fontoura e Mayer (2006), o princípio da integralidade só é possível através de um olhar atento que possibilite apreender as necessidades das ações levando em conta a contextualização. E, conforme Cecílio (2001) alerta, uma integralidade focalizada pode ser identificada no espaço bem delimitado de um serviço de saúde, referida ao caso de uma experiência vivenciada e conquistada por meio do esforço e confluência dos vários saberes de uma equipe multiprofissional.

Segundo Trad (2010), a problematização das relações familiares faz-se necessária para a atenção integral à saúde, tanto no que se refere às formas próprias de cuidado à saúde e às doenças utilizadas pelas famílias, como também no que diz respeito às implicações das relações familiares no próprio processo de adoecer, dentro do que pretende ser uma compreensão ampliada do processo saúde-doença-cuidado, que leve em conta as dimensões biológicas, psicológicas e sociais deste processo.

Infelizmente, em grande número de realidades distribuídas ao longo deste país continental, existe uma diferença entre a forma como o modelo de vigilância à saúde foi concebido, e a sua aplicação prática na Estratégia Saúde da Família, que continua sendo operacionalizado, em muitos cenários, através de um sistema fragmentado, focal, desarticulado e segmentado, padecendo das mesmas mazelas que justificaram a urgência da sua implantação no passado.

Outra barreira a ser transposta é a limitação da abordagem familiar. Falta ainda formação e vocação para esta estratégia que, embora carregue literalmente o substantivo famulus (família) na certidão de nascimento, carece de verbo (ação articulada e tempo oportuno) para aplicar uma práxis efetiva capaz de consolidar a atenção integral às famílias.

Neste contexto, a Estratégia Saúde da Família requer o desenvolvimento da capacidade técnica de identificação e hierarquização das necessidades sociais em saúde, através da organização de saberes e de instrumentos que sejam adequados para priorizar a atenção domiciliar enquanto prática indispensável ao processo de trabalho multiprofissional e interdisciplinar.

A Escala de Coelho é um instrumento de estratificação de risco familiar, desenvolvido no município de Contagem-MG, que, aplicado às famílias adscritas a uma equipe de saúde, pretende determinar seu risco social e de saúde, refletindo o potencial de adoecimento de cada núcleo familiar.

Este instrumento possui o diferencial facilitador de utilizar os dados referidos como sentinelas de risco (tabela 1), disponibilizados na ficha A do Sistema de Informação da Atenção Básica (SIAB) disponíveis na rotina das equipes de saúde.

Tabela 1: Sentinelas de Risco, Definições das Sentinelas e Escore de Risco.

\begin{tabular}{|l|c|c|}
\hline $\begin{array}{c}\text { Dados da Ficha A } \\
\text { (Sentinelas de Risco) }\end{array}$ & Definições das Sentinelas de Risco & $\begin{array}{c}\text { Escore de } \\
\text { Risco }\end{array}$ \\
\hline Acamado & $\begin{array}{c}\text { Toda pessoa restrita ao seu domicílio, por falta de } \\
\text { habilidade e/ou incapacidade de locomoção por si }\end{array}$ & 3 \\
\hline
\end{tabular}




\begin{tabular}{|c|c|c|c|}
\hline & só a qualquer unidade de saúde. & & \\
\hline Deficiência Física & $\begin{array}{l}\text { Defeito ou condição física de longa duração ou } \\
\text { permanente que dificulta ou impede a realização } \\
\text { de determinadas atividades cotidianas, escolares, } \\
\text { de trabalho ou de lazer. }\end{array}$ & & 3 \\
\hline Deficiência Mental & $\begin{array}{l}\text { Defeito ou condição mental de longa duração ou } \\
\text { permanente que dificulta ou impede a realização } \\
\text { de determinadas atividades cotidianas, escolares, } \\
\text { de trabalho ou de lazer. }\end{array}$ & & 3 \\
\hline $\begin{array}{l}\text { Baixas Condições de } \\
\text { Saneamento }\end{array}$ & $\begin{array}{l}\text { Saneamento implica no controle dos fatores do } \\
\text { meio físico do homem que podem exercer efeitos } \\
\text { prejudiciais à sua saúde. }\end{array}$ & & 3 \\
\hline Desnutrição (Grave) & $\begin{array}{l}\text { Percentil menor que } 0,1 \text { e peso muito baixo para a } \\
\text { idade. }\end{array}$ & & 3 \\
\hline Drogadição & $\begin{array}{l}\text { Utilização compulsiva de drogas lícitas ou ilícitas } \\
\text { que apresentem potencial para causar dependência } \\
\text { química (álcool, tabaco, benzodiazepínicos, } \\
\text { barbitúricos, e drogas ilícitas). }\end{array}$ & & 2 \\
\hline Desemprego & $\begin{array}{l}\text { Situação na qual a pessoa não esteja exercendo } \\
\text { nenhuma ocupação (não incluir na avaliação } \\
\text { férias, licenças ou afastamentos temporários). A } \\
\text { realização de tarefas domésticas é considerada } \\
\text { ocupação (trabalho doméstico), mesmo que não } \\
\text { seja remunerado. }\end{array}$ & & 2 \\
\hline Analfabetismo & $\begin{array}{l}\text { Pessoa que, a partir da idade escolar, não sabe ler } \\
\text { nem escrever no mínimo um bilhete, e/ou que sabe } \\
\text { apenas assinar o nome. }\end{array}$ & & 1 \\
\hline Menor de Seis Meses & Lactente com idade até 5 meses e 29 dias. & & 1 \\
\hline $\begin{array}{l}\text { Maior de Setenta } \\
\text { Anos }\end{array}$ & Toda pessoa com mais de 70 anos completos. & & 1 \\
\hline $\begin{array}{l}\text { Hipertensão Arterial } \\
\text { Sistêmica }\end{array}$ & $\begin{array}{l}\text { Pressão arterial sistólica maior ou igual a } \\
\text { 140mmHg e pressão arterial diastólica maior ou } \\
\text { igual a } 90 \mathrm{mmHg} \text {, em indivíduos que não usam } \\
\text { medicação anti-hipertensiva. }\end{array}$ & & 1 \\
\hline Diabetes Mellitus & $\begin{array}{l}\text { Grupo de doenças metabólicas caracterizadas por } \\
\text { hiperglicemia e associadas a complicações, } \\
\text { disfunções e insuficiência de vários órgãos. }\end{array}$ & & 1 \\
\hline Relação & Número de cômodos na residência dividido pelo & $>1$ & 3 \\
\hline
\end{tabular}




\begin{tabular}{|l|l|l|l|}
\hline Morador/Cômodo & $\begin{array}{l}\text { número de moradores do domicílio. São } \\
\text { considerados cômodos todos os compartimentos } \\
\text { integrantes do domicílio, inclusive banheiro e } \\
\text { cozinha, separados por paredes, e os existentes na }\end{array}$ & $<1$ & 2 \\
& $\begin{array}{l}\text { parte externa do prédio, desde que constituam } \\
\text { parte integrante do domicílio, com exceção de } \\
\text { corredores, alpendres, varandas abertas, garagens, } \\
\text { depósitos. }\end{array}$ & & \\
\hline
\end{tabular}

\section{Fonte: Modificado de Savassi et al (2012).}

A pontuação deve ser computada somando os escores a cada pessoa portadora da condição, no caso das sentinelas de caráter individual. Em áreas nas quais as famílias apresentam escore final muito elevado, recomenda-se o aumento do ponto de corte para uma melhor priorização dos casos de risco familiar mais alto.

No caso de sentinelas que acometem grandes áreas, tipo baixas condições de saneamento, a classificação desta situação passa a ser de área de risco comum às famílias e não de risco familiar singular, uma vez que envolvem aspectos que transpassam o espaço domiciliar e estão relacionados com a promoção de políticas públicas saudáveis.

As famílias, a partir da soma dos escores atribuídos, são classificadas (tabela 2) em risco menor a máximo (R1 a R3). As sentinelas de risco foram selecionadas por sua relevância epidemiológica, sanitária e pelo potencial de impacto socioeconômico na dinâmica familiar, nas relações entre os indivíduos no núcleo da família e no trabalho.

\section{Tabela 2: Cálculo do risco familiar}

\begin{tabular}{|c|c|}
\hline Escore Total & Risco Familiar \\
\hline $\mathbf{5}$ ou $\mathbf{6}$ & R1 - Risco menor \\
\hline 7 ou 8 & R2 - Risco médio \\
\hline Acima de 9 & $\mathbf{R 3}-$ Risco máximo \\
\hline
\end{tabular}

Fonte: Savassi et al (2012).

Coelho e Savassi (2004) indicam que existem duas formas de visita: a visita domiciliar Fim, com objetivos específicos de atuação (propaganda política, internação domiciliar, atenção domiciliar terapêutica, visita a pacientes acamados, na atenção ao parto); e a visita domiciliar Meio, voltada para a abordagem estratégica da família (busca ativa em demanda reprimida, educação para a saúde mais individualizada, estímulo ao cuidado com a saúde, apontamento de necessidades para ações de promoção à saúde, e no estabelecimento de canais permanentes de comunicação). 
Savassi et al (2012) advertem que esta escala não foi desenvolvida para a finalidade de abordagem familiar, nem para classificar a totalidade dos riscos individuais e muito menos não envolve os diversos riscos presentes em uma família, porém serve de instrumento auxiliar para a avaliação da vulnerabilidade familiar e como mais uma ferramenta para planejamento das ações da equipe, pois serve de ponto de partida para um mapeamento das redes sociais de apoio ao núcleo familiar.

\section{CAMINHO METODOLÓGICO}

Trata-se de um estudo metodológico de validação de um instrumento denominado Escala de Risco Familiar de Coelho e Savassi, aplicado para definir as prioridades de visita domiciliar. Os dados utilizados foram obtidos através das fichas A, de acompanhamento do Agente Comunitário de Saúde. As fichas que não estavam preenchidas corretamente ou em branco foram oportunamente atualizadas. Foram Também utilizados registros de observações em diário de campo e dos portfólios de aprendizagem dos alunos dos cursos da área de saúde da UFRN.

O local do estudo foi a Unidade de Saúde da Família de Cidade Praia, escolhida por ser campo de prática dos discentes bolsistas e não bolsistas do Programa de Educação Tutorial na Saúde (PET-Saúde) e esta estratégia foi viabilizada através da articulação entre o Mestrado Profissional em Saúde da Família (RENASF/UFRN), e a preceptoria das disciplinas Atividade Integrada de Educação Saúde e Cidadania (SACI) e no Programa de Orientação Tutorial para o Trabalho Integrado em Saúde (POTI).

Essas disciplinas são ofertadas no primeiro ano dos cursos da saúde e proporcionam aos participantes uma maior articulação entre a teoria e a prática na direção da aprendizagem significativa ao trabalhar temas pertinentes referentes à territorializaçãoe mapeamento da área de abrangência, ação multiprofissional em domicílio, visitas aos equipamentos sociais,notificação e o registro deinformações epidemiológicas, enfatizando a incubação de tecnologia leves que possam produzir melhoria na qualidade de vida da comunidade.

A Unidade de Saúde da Família de Cidade Praia foi implantada em 2002, no Distrito Sanitário Norte I (Natal-RN), comporta duas equipes de saúde da família. Cada equipe (046 e 048) contém dentista, médico, enfermeiro, dois técnicos de enfermagem, um técnico em saúde bucal e cinco a seis agentes comunitários de saúde. Este quadro funcional é complementado pela gerência local (Diretor e Administrador), por um agente de serviços gerais (terceirizado) e cinco agentes patrimoniais.

A unidade está assessorada pelo Núcleo de Apoio à Saúde da Família (NASF) que possui os profissionais: Educador Físico, Assistente Social, Fisioterapeuta, Psicólogo, Farmacêutico e Nutricionista. Este suporte foi fundamental para a promoção da qualidade dos debates nos grupos de discussão.

Seu território de abrangência é predominantemente residencial onde uma parte de seus domicílios pertence a um conjunto habitacional (micro áreas 01 e 03 da equipe 046 e micro áreas 02 e 03 da equipe 048), e a outra parte é composta por loteamentos (demais micro áreas). Tem uma população predominante de adultos jovens, e não dispõe de equipamentos comunitários de lazer, esportes ou de convivência.

A unidade procurou, desde sua implantação, organizar as agendas de atenção à saúde por ciclo de vida através da prática do acolhimento, procurando romper com a lógica das agendas fechadas e pré-definidas, buscando redirecionar a demanda espontânea para as 
atividades organizadas para uma oferta programada. Durante o acolhimento, o usuário é convidado a participar nos grupos que estiverem constituídos naquele momento.

Desde o ano de 2009 a unidade de saúde vem sendo palco da introdução precoce dos alunos de graduação de diversos cursos da área de saúde aos cenários reais dos serviços municipais de atenção básica orientados pelas diretrizes da Estratégia Saúde da Família, para que os discentes possam compreender e enfrentar, em um ambiente democrático, os diversos fatores relacionados com a produção e determinação do processo saúde e doença.

O estudo foi realizado durante o primeiro semestre letivo do ano de 2013, em três etapas. $\mathrm{Na}$ fase inicial ocorreram rodas de leitura para esclarecimento sobre as condições sentinelas, conhecimento e debate sobre o tema visita domiciliar com enfoque no instrumento (escala) de estratificação de risco a ser utilizado (proposto por Coelho e Savassi). Participaram naquele momento os discentes dos cursos de saúde da Universidade Federal do Rio Grande do Norte (UFRN), o Grupo Tutorial do PET-Saúde Cidade Praia, os profissionais do NASF, e todos os Agentes Comunitários de Saúde da unidade.

Na segunda etapafoi feita a classificação das famílias cadastradas na unidade de saúde, categorizando-as em riscos R1, R2 e R3 (tabelas 3 e 4). A escala foi aplicada em regime de mutirão através de grupos de trabalho compostos por Agentes Comunitários de Saúde e Monitores, sempre supervisionados por Preceptores. Esta etapa foi realizada a partir da análise de 1904 fichas A do Sistema de Informação da Atenção Básica (SIAB), que são preenchidas pelos Agentes Comunitários de Saúde (ACS) na primeira visita às famílias.

A escolha desta ficha, feita pelos idealizadores da escala, resultou de sua objetividade em apresentar os dados fundamentais para se estabelecer um corte dinâmico da população que se pretende abordar. Desta forma as informações recolhidas permitem às Equipes de Saúde da Família o conhecimento dos indicadores demográficos, socioeconômicos e de morbimortalidade referidos nas famílias das suas áreas de abrangência, requisito fundamental para realização do planejamento estratégico local.

Por fim, a terceira etapa (final) contemplou a apresentação e discussão dos resultados obtidos, por um grupo de atores chaves composto por tutor, preceptores, profissionais de saúde das equipes e do NASF e a gestão local para validação do instrumento e socialização dos aspectos pertinentes decorrentes do percurso e os limites da aplicação da escala na realidade observada. Após este momento foram realizadas visitas domiciliares às famílias de grau máximo (R3) em ordem decrescente para observação in loco dos critérios de classificação.

\section{RESULTADOS E DISCUSSÃO}

A situação de risco individual e social de uma família pode ser entendida como a circunstância vivida por este coletivo quando está determinada pelas características gerais dos diversos problemas que lhe conferem desvantagens nas múltiplas inter-relações de casualidade, determinações e de iniquidades do cotidiano.

A utilização da Escala representou a incorporação de um instrumento básico no cotidiano do planejamento das ações, pela eleição de informações como sentinelas para avaliação das situações a que as famílias possam estar expostas no dia-a-dia.Foram analisadas 1904 famílias: $1622(85,19 \%)$ categorizadas como R1, 164 (8,61\%) classificadas como R2 e 118 $(6,2 \%)$ consolidadas como R3. 
Em uma vista panorâmica os resultados foram semelhantes para as duas equipes, mostrando uma fronteira estreita entre os escores de risco médio e elevado, o que denota a necessidade de atualização constante no consolidado desses dados, pela possibilidade de migração de famílias do escore R2 para R3 e vice versa, frente à dinâmica do território vivo.

Com isso foi verificada uma quantidade significante de famílias que necessitam de uma maior atenção por parte dos profissionais de saúde, justamente as que demandam o maior número de visitas domiciliares e demais serviços de saúde ofertados, uma vez que os escores médio e alto estiveram muito próximos.

Observando atentamente os resultados para cada equipe em particular foram evidenciadas algumas características singulares, o que justifica a pertinência do instrumento quanto à sua aplicação em cada estrato de território. Verificamos que dentro de uma mesma equipe as micro áreas possuem diferentes avaliações de risco, embora a proximidade entre as famílias de risco R2 e risco R3 seja confirmada.

Essas semelhanças e divergências merecem ser analisadas com cautela, inclusive algumas micro áreas foram revisadas para afastamento do viés do sub registro. A diversidade quanto aos graus de riscodemonstraram a heterogeneidade presente em micro áreas aparentemente homogêneas.A equipe 046 teve 1049 famílias analisadas, sendo que 902 (86\%) foram classificadas como R1, 86 (8,20\%) obtiveram R2 e $61(5,80 \%)$ R3.

Nesta equipe (ver tabela 3) as micro áreas pertencentes a loteamentos (ruas sem pavimentação e com água servida escoando em valas a céu aberto) mostraram resultados próximos àquelas formadas por conjuntos habitacionais (ruas pavimentadas e com calçadas).

Tabela 3: Escores (Números absolutos e porcentagem) da Escala de Coelho, por micro áreas Equipe 046 na Unidade de Saúde da Família de Cidade Praia.

\begin{tabular}{|c|c|c|c|c|c|c|c|c|c|c|c|c|}
\hline \multicolumn{1}{|c|}{ ESCALA DE COELHO E SAVASSI - USF CIDADE PRAIA } \\
\hline $\begin{array}{c}\text { EQUIPE } \\
\text { 046 }\end{array}$ & \multicolumn{2}{|c|}{ MICRO 01 } & \multicolumn{2}{|l|}{ MICRO 02 } & \multicolumn{2}{l|}{ MICRO 03 } & \multicolumn{2}{l|}{ MICRO 04 } & \multicolumn{2}{l|}{ MICRO 05 } & \multicolumn{2}{l|}{ MICRO 06 } \\
\cline { 2 - 14 } & № & $\%$ & № & $\%$ & № & $\%$ & № & $\%$ & № & $\%$ & № & $\%$ \\
\hline R1 & 164 & 91,62 & 126 & 86,9 & 132 & 81,89 & 158 & 85,87 & 136 & 79,54 & 196 & 88,69 \\
\hline R2 & 11 & 6,15 & 10 & 6,9 & 15 & 10,06 & 16 & 8,69 & 20 & 11,69 & 14 & 6,33 \\
\hline R3 & 4 & 2,23 & 9 & 6,2 & 12 & 8,05 & 10 & 5,44 & 15 & 8,77 & 11 & 4,98 \\
\hline TOTAL & 179 & 100 & 145 & 100 & 149 & 100 & 184 & 100 & 171 & 100 & 221 & 100 \\
\hline
\end{tabular}

Por sua vez, a equipe 048 (vide tabela 4) apresentou duas micro áreas em que o escore R3 foi maior do que o R2, e curiosamente mostrou uma maior divergência entre as áreas de conjuntos habitacionais (micro áreas 01 e 02) e de loteamentos (micro áreas 04 e 05), 
entretanto a micro área 03, que é exclusivamente de conjunto habitacional, apresentou um resultado semelhante às micro áreas de loteamento. Nesta equipe $720(84,20 \%)$ famílias obtiveram a classificação R1, $78(9,10 \%)$ R2 e $57(6,70 \%)$ R3, de um total de 855 domicílios.

Tabela 4: Escores (Números absolutos e porcentagem) da Escala de Coelho, por micro áreas Equipe 048 na Unidade de Saúde da Família de Cidade Praia.

\begin{tabular}{|c|c|c|c|c|c|c|c|c|c|c|}
\hline \multicolumn{8}{|c|}{ ESCALA DE COELHO E SAVASSI - USF CIDADE PRAIA } \\
\hline \multirow{2}{*}{ EQUIPE 048 } & MICRO 01 & \multicolumn{2}{l|}{ MICRO 02 } & \multicolumn{2}{l|}{ MICRO 03 } & \multicolumn{2}{l|}{ MICRO 04 } & \multicolumn{2}{l|}{ MICRO 05 } \\
\cline { 2 - 12 } & № & $\%$ & № & $\%$ & № & $\%$ & № & $\%$ & № & $\%$ \\
\hline R1 & 154 & 90,58 & 139 & 86,87 & 109 & 78,98 & 144 & 78,69 & 174 & 85,30 \\
\hline R2 & 14 & 8,24 & 15 & 9,38 & 13 & 9,42 & 23 & 12,57 & 13 & 6,37 \\
\hline R3 & 2 & 1,18 & 6 & 3,75 & 16 & 11,6 & 16 & 8,74 & 17 & 8,33 \\
\hline TOTAL & 170 & 100 & 160 & 100 & 138 & 100 & 183 & 100 & 204 & 100 \\
\hline
\end{tabular}

Nas duas equipes de saúde da família em que foi aplicada, a escala de risco familiar de Coelho-Savassi foi útil na reorganização da demanda ao promover uma percepção mais apurada, objetiva e quantificada do risco das famílias avaliadas, impactando de maneira positiva o trabalho em equipe.

A partir de perfis divergentes é possível traçar prioridades fundamentadas no princípio da equidade (tratar de forma diversificada os diferentes) para ampliar a capacidade de mobilização dos recursos disponíveis de acordo com as necessidades identificadas, inclusive abrindo possibilidades para aplicação de ferramentas de abordagem familiar (tipo Genograma, Ecomapa, P.R.A.C.T.I.C.E., F.I.R.O.) que possam complementar as ações coordenadas.

Entretanto é importante um maior investimento em outros instrumentos de avaliação e análise das necessidades e ações de saúde, assim como o desenvolvimento de estratégias para uma priorização que envolva a consolidação do diálogo entre os diferentes profissionais da equipe, para criação de um espaço de discussão e definição de prioridades a partir das reais necessidades de saúde dos indivíduos e do seu coletivo.

Nesse sentido, concordamos com Ayres (2003) quando aponta a vulnerabilidade como conceito estruturante das abordagens de avaliação das condições de vida e saúde fundamentada na atribuição de sentidos e na interpretaçãoda pluralidade dinâmica e complexa dos fenômenos sociais, inconstantes e relativos.

Nascimento et al (2010) apontam alguns critérios que mereciam um esforço de inclusão no rol das sentinelas de acompanhamento, tais quais as precárias condições de higiene pessoal, a exposição a agentes poluidores domésticos, a presença de alergenos 
constantemente inalados, bem como alguns aspectos tipo desemprego, sedentarismo, condutas pessoais de alimentação, desmotivações para adesão às condutas terapêuticas, indisposição para realização de atividades físicas e cultura do movimento e dimensões interpessoais e criminais.

É pertinente ultrapassar o enfoque de risco, de característica predominantemente mecânica, em busca de novas formas de análises que possam estar mais condizentes com a realidade particular de cada cenário permeados pela teia de relações vivenciadas nos espaços de construção social e convivência entre os serviços de saúde e os grupos sociais.

Em relação aos participantes discentes dos cursos da área de saúde, visualizamos com certa expectativa um impacto nas suas trajetórias acadêmicas, pela ação transformadora da produção, inovação e transferência de conhecimentos diante da ampliação de oportunidades educacionais durante o processo de formação e de qualificação.

Essa possibilidade de transformação está em consonância com a adequação do cenário da prática ao método pedagógico da problematização, através de rodas democráticas de conversação, compartilhando os conteúdos para fazer da experiência também uma ação educativa para compreender a realidade.

A experimentação de alternativas metodológicas de ensino, pesquisa e extensão vêm contribuindo no processo da preparação desses alunos para sua futura atuação profissional, inclusive pelo caráter multidisciplinar da atividade proposta, e pela alternância dos lugares tradicionalmente reservados para os discentes, docentes, preceptores e profissionais de saúde.

A oferta de espaços de escuta e reflexão para aperfeiçoamento da consciência crítica de si e do outro, torna o próprio processo educativo participante uma grande lição de sabedorias práticas, ampliando o ambiente de ensino-aprendizagem para além da metragem reduzida de uma sala de aula, em direção à realidade concreta.

\section{CONSIDERAÇÕES FINAIS}

Finalizando, evidenciamos que a partir do uso da escala proposta por Coelho e Savassi para estratificação de risco familiar, foi possível estabelecer estratégias para priorizar as visitas domiciliares nas micro áreas de maior necessidade, de uma maneira simples, fácil e clara utilizando um formulário tradicionalmente presente no cotidiano das equipes (a ficha A do SIAB) não demandando a elaboração de outro documento para esta finalidade.

Sugerimos a realização de estudos complementares para aferição do grau de divergência na classificação de risco feita por cada profissional avaliador, bem como a premissa de que esta avaliação seja feita em grupos multiprofissionais para minimização do viés da descalibração e ampliação dos olhares sócios sanitários.

As famílias que possuem nível de educação mais baixo e recursos financeiros mais limitados tendem a apresentar maior dificuldade para frequentar círculos sociais mais variados. Assim, a diminuição deste multipertencimento se torna uma consequência natural, uma vez que circuitos sociais mais restritos determinam situações mais singulares relativos aos papéis familiares, à comunicação e socialização, que podem ser peculiares a respeito da determinação do risco pessoal e social.

Há de se ter cuidado com as necessidades de saúde consideradas de risco baixo (R1). A inércia de ações focadas nas famílias de risco mínimo poderá elevar a classificação desta 
graduação de risco, no futuro, para os níveis médio ou máximo, pois essas famílias exigem menos da assistência curativa e/ou reabilitadora, entretanto merecem maior atenção quanto aos aspectos de promoção e prevenção.

Visualizamos ainda que a escala de Coelho e Savassi necessita de uma readequação após a chegada das novas fichas de cadastro familiar e individual, que gradativamente substituirão as fichas A, propostas pelo Sistema de Informação E-SUS. É pertinente uma revisão criteriosa de todos os aspectos inclusive em relação à inclusão de novas condições sentinelas anteriormente não contempladas.

\section{REFERÊNCIAS BIBLIOGRÁFICAS}

1. AYRES JRCM, FRANÇA J.I., CALAZANS G.J., SALETTI H.C. O conceito de vulnerabilidade e as práticas de saúde: novas perspectivas e desafios. In: CZERESNIA D, FREITAS CM. (Org.). Promoção da saúde: conceitos, reflexões, tendências. Rio de Janeiro: Fiocruz; 2003.p.117-139.

2. AYRES, J.R.C.M. et al. Caminhos da integralidade: adolescentes e jovens na Atenção Primária à Saúde. Interface comunicação saúde educação v.16, n.40, p.67-81, jan./mar. 2012.

3. BASTOS, A.C.S. \& FRANCO, A.L.S. Aspectos da interação do médico com a família. In: LEITE et al. Habilidades de comunicação com pacientes e famílias. São Paulo: Editora Sarvier, 2007. 242p.

4. BRASIL, Ministério da Saúde. SIAB: manual do sistema de Informação de Atenção Básica. Brasília: Ministério da Saúde, 2003. 96 p. (Série A. Normas e Manuais Técnicos).

5. CECÍLIO, L. C. O. As necessidades de saúde como conceito estruturante na luta pela integralidade e equidade na atenção em saúde. In: PINHEIRO, R., MATTOS, R. A. (Orgs.) Os sentidos da integralidade na atenção e no cuidado de saúde. Rio de Janeiro: IMS-UERJ/Abrasco, 2001.p.113-126.

6. CHAPADEIRO, Cibele Alves et al. A família como foco da atenção primária à saúde. Belo Horizonte: Nescon/UFMG, 2011. SOUZA, Alex Sander Ribas. MENDES, Artur Oliveira. Abordagem Familiar. Associação Mineira de Medicina de Família e Comunidade. Grupo de Estudos em Saúde da Família, 02/04/2007. Disponível em: http://www.smmfc.org.br/mfcaz/gesfabordagemmarcoteorico.pdf.

7. COELHO, Flávio; SAVASSI, Leonardo. Aplicação de escala de risco familiar como instrumento de organização das visitas domiciliares. RBMFC, vol. 1, número 2, p.1926.

8. FONTOURA, R.T. \& Mayer, C.N. Uma breve reflexão sobre a integralidade. RevBrasEnferm 2006 jul-ago; 59(4): 532-7. 
9. NASCIMENTO F.G. et al. Aplicabilidade de uma escala de risco para organização do processo de trabalho com famílias atendidas na Unidade Saúde da Família em Vitória (ES). Ciência \& Saúde Coletiva, 15(5):2465-2472, 2010.

10. SAVASSI, Leonardo; LAGE, Joana; COELHO, Flávio. Sistematização de um instrumento de estratificação de risco familiar: Escala de risco familiar de CoelhoSavassi. J ManagPrim Health Care 2012; 3(2):179-185.

11. SILVA, Janaina Vieira da. SANTOS, Sueli Maria dos Reis. Trabalhando com Famílias Utilizando Ferramentas. Revista APS, v.6, n.2, p.77-86, jul./dez. 2003.

12. TRAD, L. A. B. (Org.). Família contemporânea e saúde. Significados, práticas e políticas públicas. Rio de Janeiro: Editora Fiocruz, 2010. 